\title{
CURADORIA DIGITAL DE DADOS CIENTÍFICOS: PELO VIÉS DE UM PERIÓDICO
}

\author{
Claudiane Weber \\ Doutoranda em Ciência da Informação pela Escola de Comunicações e Artes da Universidade de São Paulo (USP).
}

\begin{abstract}
Resumo
O presente estudo analisa a produção sobre a curadoria digital através de um recorte realizado sobre o assunto no periódico International Journal of Digital Curation. Por meio de análise de conteúdo, foram pesquisados os resumos de 220 artigos, no período que vai desde a criação do periódico em 2006, até dezembro de 2013. Quanto à apreciação dos registros levantados, o foco da investigação foi voltado para busca de como é entendido, e, ao o que está relacionado o tema curadoria digital de dados científicos. Como resultado, identificamos aproximadamente 360 autores e coautores. Os resumos apresentaram diferentes enfoques, como a curadoria de dados, o papel do curador científico, a preservação digital, a reutilização de dados científicos e a e-Science.
\end{abstract}

\section{Palavras-chave}

Curadoria de dados. Curador. E-Science.

\section{DIGITAL CURATION OF SCIENTIFIC DATA ACCORDING TO A JOURNAL'S BIAS}

\begin{abstract}
This study examines the production of digital curation across a clipping on the subject in the International Journal of Digital Curation. Through content analysis, were searched abstracts of 220 articles, in the period since the inception of the journal in 2006 to December 2013. Assessment of the textual part of the records collected, the focus of the investigation was how it's understood, and about what is related the subject digital curation of scientific data. As a result, we identified approximately 360 authors and co-authors. Abstracts presented different approaches, such as data curation, the role of scientific curator, digital preservation, reuse scientific data and e-Science.
\end{abstract}

\section{Keywords}

Data Curation. Curator. E-Science.

\section{INTRODUÇÃO}

Para efetivar as reflexões sobre a literatura de um campo do conhecimento faz-se necessário pensar nas diversas possibilidades e suportes dessa literatura. Dentro do ciclo produtivo da ciência, o periódico científico se mantém como o principal veículo de comunicação formal dos resultados 
de pesquisas originais. E ao estar vinculado a uma organização editorial, torna-se o instrumental que reproduz as sanções e exigências próprias do campo científico, aponta o grau de evolução de cada área de conhecimento, estabelece a propriedade intelectual e legitima novos campos de estudos.

A análise das revistas científicas, segundo Bufrem et. al. (2007, p. 39) "tem sido uma modalidade de estudo com presença significativa e reiterada na literatura voltada à produção de conhecimento." Ainda para estes autores, esse fenômeno é associado à necessidade sentida pelos pesquisadores por fontes informacionais para o domínio da literatura de sua área e para a difusão de suas próprias pesquisas. E, além do mais, a publicação científica tornou-se um instrumento para a promoção e fortalecimento do ciclo de criação, organização e difusão das informações, influenciando no ritmo da produção do conhecimento.

Sabendo da grande importância da análise de periódicos para uma área, associados à necessidade sentida por fontes informacionais para o domínio da literatura de um tema, a presente pesquisa pretende apresentar um breve panorama do conceito de curadoria digital a partir de um recorte efetuado num periódico específico da área.

A curadoria digital emerge como um campo de pesquisa e prática interdisciplinar que reflete uma abordagem holística para o gerenciamento do objeto digital. Para Sayão e Sales (2012, p.189), a curadoria digital surge como uma nova área de práticas e de pesquisa de espectro amplo que dialoga com várias disciplinas e diversos gêneros de profissionais. "Isto porque, como se trata de uma área que só recentemente despontou como crítica para a pesquisa, ainda restam muitas lacunas práticas e teóricas a serem equacionadas, orientadas, preferencialmente, por uma abordagem multidisciplinar", ressaltam os autores.

Deste modo, a pesquisa buscou verificar como se apresenta a curadoria digital pelo viés de um periódico. Como ponto de partida e, na busca de respostas, optamos por um levantamento em um periódico que traz no título o termo "curadoria digital”, assim o International Journal of Digital Curation $^{1}$ foi o selecionado para essa pesquisa. E, para tal, formalizamos a questão: o que tem sido escrito sobre a curadoria digital num periódico científico ao longo do tempo? Definimos como objetivo geral verificar o que é a curadoria digital no periódico International Journal of Digital Curation, e a que está relacionado o tema nessa publicação.

\section{METODOLOGIA}

${ }^{1}$ Disponível em: http://www.ijdc.net/index.php/ijdc/index 
Para investigar a produção sobre o tema foi realizado um levantamento no periódico International Journal of Digital Curation. O período de abrangência foi desde a criação do periódico em 2006, até a publicação do último fascículo em dezembro de 2013.

A partir de amostra representativa de artigos sobre o tema, avaliamos todos os títulos e resumos. Fez-se análise de características relativas aos documentos como autoria, afiliação dos autores, ano de publicação, idioma. Não avaliamos palavras-chave, pois neste periódico elas são ausentes do resumo. .

A análise foi iniciada a partir de uma "leitura flutuante" dos resumos dos artigos. É denominado "leitura flutuante" o primeiro contato do analista com os documentos em estudo, que visaobter "impressões e orientações" a respeito dos mesmos (BARDIN, 1977).

Ao longo desse processo de leitura, foram selecionados os resumos que apresentaram maior proximadade com o objetivo da pesquisa. Esses serviram de base para a elaboração de uma fichapadrão para a análise individual de cada resumo. Uma vez organizados, os resumos selecionados foram submetidos a uma análise de conteúdo detalhada. A análise de conteúdo é definida como uma técnica de tratamento de dados de pesquisa voltada para uma descrição objetiva, sistemática e quantitativa do conteúdo. Essa técnica ainda que tenha suas origens na pesquisa quantitativa, busca a interpretação de materiais de caráter qualitativo (MINAYO, 1998).

O levantamento estatístico quantitativo em relação à autoria aconteceu de forma aproximada, por não termos acesso completo à base de dados do periódico e pelo fato do levantamento dessa informação no próprio site da revista estar comprometido, pela inconsistência de alguns dados.

A amostragem, para o presente estudo, consitiu no levantamento e estudo dos títulos e resumosde todos os 220 artigos publicados, de aproximadamente 360 autores e coautores.

\section{RESULTADOS}

\subsection{O periódico}

O International Journal of Digital Curation (IJDC) ${ }^{2}$ é publicado pelo Centro de Curadoria Digital $^{3}$ da Universidade de Edimburgo (UK) e também está vinculado à Universidade de Bath

\footnotetext{
${ }^{2}$ http://www.ijdc.net/index.php/ijdc/about/contact

${ }^{3}$ DIGITAL CURATION CENTRE. Disponível em: < http://www.dcc.ac.uk/ > Acesso em: 03 jun. 2014.
} 
(UK). De acordo com seu site, é uma revista internacional comprometida com a excelência acadêmica e dedicada ao avanço de curadoria digital em uma ampla gama de setores. Sua publicação é feita somente na língua inglesa com periodicidade semestral. Seu conselho editorial é internacional e eminente para informar e orientar sobre o tema.

O IJDC, com base em suas diretrizes editoriais, publica dois principais tipos de submissão: artigos revisados por pares (peer-reviewed) e artigos em geral.

Um artigo peer-reviewed deve ser original e inovador. Deve incluir ou fazer referência à evidência significativa (seja experimental, observacional ou textual) para apoiar as conclusões.

A sessão de artigo geral é descritiva de algum evento relevante, atividade, projeto de pesquisa em andamento. Pode se apresentar uma proposta de uma aplicação, um serviço, ou um projeto, por exemplo. Artigos gerais também podem ser artigos de opinião, comentários, ou levantamentos do trabalho existente em alguma área particular.

\subsection{Autores}

Todo trabalho científico é oriundo de uma ideia, na maioria das vezes buscando resposta para um problema. A principal condição para ser incluído entre os autores de um trabalho científico é ter participado intelectualmente na elaboração, análise ou redação do trabalho. Assim, não poderíamos deixar de levantar as autorias desses 220 artigos publicados, bem como a sua afiliação (se vinculado a alguma universidades), e a naturalidade.

No levantamento realizado identificamos aproximadamente 360 autores e coautores, destes, em torno de $95 \%$ oriundos de países de língua inglesa. Com maior destaque para Grã-Bretanha (Inglaterra e Escócia) seguido pelos Estados Unidos e por fim Canadá e Austrália. Outros países que também apresentam uma média de 3 artigos ou mais, são a Alemanha, Itália, Grécia, Finlândia, Holanda, Nova Zelândia.

No Quadro 1, trouxemos um ranking das instituições com o devido número de Autores vinculados a estas instituições e que publicaram sobre o tema curadoria. 
Quadro1: Identificação de autoria e instituição

\begin{tabular}{|c|c|c|c|}
\hline & INSTITUIÇÃO & $\begin{array}{c}\mathbf{N}^{\circ} \text { DE } \\
\text { AUTORES }\end{array}$ & VINCULAÇÃO \\
\hline 1 & $\begin{array}{l}\text { University of Edinburgh and Digital } \\
\text { Curation Centre }\end{array}$ & 34 & $\begin{array}{l}\text { Digital Curation Centre; Standards Advisor } \\
\text { DCC;Project Manager, StORe; eScience Liaison } \\
\text { Support Officer; Division of Psychiatry, School } \\
\text { of Molecular and Clinical Medicine;e-Science } \\
\text { Institute; School of Informatics;National e- } \\
\text { Science Centre; School of Informatics; Science } \\
\text { and Technology Facilities Council (formerly } \\
\text { CCLRC). }\end{array}$ \\
\hline 2 & $\begin{array}{l}\text { University of California (Berkeley, } \\
\text { Los Angeles, San Diego,etc) }\end{array}$ & 22 & $\begin{array}{l}\text { California Digital Library; UC San Diego; } \\
\text { Associate Director-Digital Curation } \\
\text { Centre;Environmental Science, Policy, and } \\
\text { Management;Department of Computer Science } \\
\text { and Genome Center; Graduate School of } \\
\text { Education and Information Studies; Scripps } \\
\text { Institution of Oceanography. }\end{array}$ \\
\hline 3 & Universityof Glasgow & 17 & $\begin{array}{l}\text { Digital Curation Centre \& Humanities Advanced } \\
\text { Technology and Information Institute; HATII; } \\
\text { School of Physics and Astronomy. }\end{array}$ \\
\hline 4 & Indiana University & 13 & $\begin{array}{l}\text { Department of Computer Science; Indiana } \\
\text { University School of Informatics and Computing }\end{array}$ \\
\hline 5 & University of Bath, UK & 12 & $\begin{array}{l}\text { UKOLN; Digital Curation Centre, eBank-UK } \\
\text { Project and KIM Project, UKOLN; } \\
\text { Research Data Scientist; Department of } \\
\text { Mechanical Engineering; } \\
\text { KIM Project;IdMRC }\end{array}$ \\
\hline & INSTITUIÇÃO & $\begin{array}{c}\mathbf{N}^{\circ} \text { DE } \\
\text { AUTORES }\end{array}$ & VINCULAÇÃO \\
\hline 6 & University of Southampton & 12 & $\begin{array}{l}\text { School of Electronics and Computer Science; IT } \\
\text { Innovation Centre; } \\
\text { Faculty of Engineering and the Environment. }\end{array}$ \\
\hline 7 & $\begin{array}{l}\text { University of Illinois at Urbana- } \\
\text { Champaign }\end{array}$ & 9 & $\begin{array}{l}\text { Graduate School of Library and Information } \\
\text { Science;Assistant University Archivist and } \\
\text { Associate Professor of Library Administration; } \\
\text { National Center for Supercomputing } \\
\text { Applications; }\end{array}$ \\
\hline 8 & King's College London & 8 & $\begin{array}{l}\text { Digital Curation Specialist, Centre for e- } \\
\text { Research; Department of Informatics, Division of } \\
\text { Health and Social Care Research; }\end{array}$ \\
\hline 9 & The British Library & 6 & $\begin{array}{l}\text { PLANETS Project Manager; Head of Digital } \\
\text { Library Technology. }\end{array}$ \\
\hline
\end{tabular}




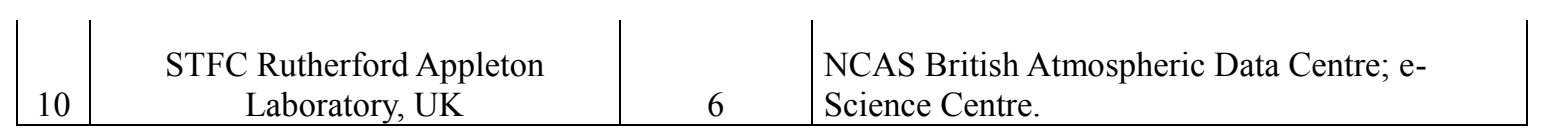

Fonte: elaborado pelas autoras em base aos dados da pesquisa (2014).

Elaboramos o quadro de acordo com a identificação dos autores nos artigos. Assim, há também uma coluna com o levantamento onde o autor identifica o local de trabalho e em alguns casos a função que exerce.

Vemos, portanto, que a Universidade de Edimburgo (Universityof Edinburgh), é a instituição com o maior número de autores que publicaram no periódico IJDC. Este número se justifica uma vez que é também a instituição que abriga o Centro de Curadoria Digital (Digital Curation Center).

Outro aspecto que devemos levar em consideração, é quanto ao número de artigos e de autores que mais contribuíram com as edições. Assim, esse ranking não é o mesmo.

As instituições que mais contribuíram com artigos foram:

1. University of Edinburgh and Digital Curation Centre;

2. University of Glasgow;

3. University of Bath.

\subsection{A curadoria}

No editorial da primeira edição de 2006, os editores afirmam que o surgimento da e-Science, onde a atividade de pesquisa está apoiada por uma computação distribuída de alto desempenho e orientada a dados, está mudando radicalmente o modo como a ciência está sendo concebida, executada e compreendida.

A criação é o financiamento, por parte do Reino Unido, do Centro de Curadoria Digital sendo um indicador da importância atribuída a estes objetivos, e o compromisso do governo com a gestão pró-ativa do registro científico para a próxima geração de pesquisadores.

O International Journal of Digital Curation surgiu diretamente a partir desta iniciativa, mas está posicionada para ser de relevância para a comunidade em geral, onde a curadoria e conservação de bens digitais é uma questão fundamental.

Também na primeira edição de 2006, destacamos o artigo de Beagrie (2006) "Digital Curation for Science, Digital Libraries, and Individuals". Para o autor o termo curadoria digital é cada vez mais utilizado para as ações necessárias para agregar valor e manter os ativos digitais ao 
longo do tempo para as gerações atuais e futuras. $\mathrm{O}$ artigo explora esse campo emergente de curadoria digital como uma área de pesquisa e prática interdisciplinar e as tendências que estão influenciando o seu desenvolvimento. Ele analisa a gênese do termo e como os papéis tradicionais relacionados com ativos digitais estão em transição. Ainda nessa edição temos um artigo que vincula a curadoria digital com a propriedade intelectual e outro que aborda a curadoria de e-mails.

A segunda edição, de 2007, foi lançada como resultado aos anais da Segunda Conferência Internacional de Curadoria Digital, realizado em novembro de 2006 em Glasgow, Escócia.

Nessa edição, a curadoria digital está amplamente vinculada à preservação digital e seus custos. Para se compreender e comunicar o custo e o valor das atividades de curadoria digital, classificadas como um fator muito importante, medidas são necessárias para garantir a sobrevivência em longo prazo de ativos digitais. Assim, projetos estão sendo desenvolvidos, bem como modelos de custeio para a preservação digital, mas ainda há um grande problema com os ativos de informação (digital ou não) em que o seu valor é difícil de expressar em termos que são facilmente compreendidos por todos os interessados, especialmente aqueles que podem financiar a sua preservação.

Destacamos, pela importância do evento, o relato de Day (2007) que traz o relatório da DigCCurr 2007, Simpósio Internacional de Curadoria Digital (Digital Curation Curriculum) realizada na Universidade da Carolina do Norte, em Chapel Hill, em 18-20 abril de 2007. O evento foi organizado como parte do projeto "Preserving Access to Our Digital Future: Buildingan International Digital Curation Curriculum", financiado pelo Instituto de Museus e Serviços de Biblioteca (IMLS). Esse simpósio se repete nos anos 2009 e 2011 e há também relatos tratando sobre essas edições.

Questionávamos, a princípio, se o termo seria - curadoria digital ou curadoria de dados? O artigo de Lambert et. al. (2007), "Data Curation Standards and Social Science Occupational Information Resources" traz no título a questão da curadoria de dados nas Ciências Sociais. No entanto, percebe-se um impasse, quando no resumo ao invés de tratar como curadoria de dados, os autores tratam de curadoria digital.

A outra edição de 2007 aborda problemas de armazenamento de dados científicos. Em especial dois artigos que tratam de dados da Astronomia e da Biologia. A preocupação está no isolamento desses dados, uma vez que eles fazem parte de um conjunto maior de informações científicas. Choudhury et al. (2007) no artigo "Digital Data Preservation for Scholarly 
Publications in Astronomy" relata sobre o início de um projeto cujo objetivo é a implementação de um protótipo de sistema, que, através de uma parceria entre sociedade, editores e bibliotecas de pesquisa, com o objetivo de estabelecer uma rede distribuída de curadoria e de repositórios de dados permanentes.

Sarah Higgins é uma das autoras mais destacadas quando o assunto é curadoria digital. Ela traz um artigo, na edição de 2008, sobre o "The DCC Curation Lifecycle Model." Esse modelo é expresso graficamente e para transmitir essa ideia de presença contínua, essas ações estão representadas como anéis concêntricos envolvendo os objetos de dados que estão no centro do modelo. O gerenciamento do ciclo de vida dos materiais digitais pode ajudar com a conceituação das etapas necessárias para a curadoria com sucesso. O modelo do projeto genérico do DCC identifica ações de curadoria aplicáveis quer em todo o ciclo de vida digital ou sequencialmente ao longo dela (HIGGINS, 2008).

Para Pryor (2007), "Some Challenges for eScience Liaison”, os desafios para a e-Science, são a promoção de conhecimentos e boas práticas de curadoria digital de dados, e não são um mero exercício de teoria. Através de sua nova iniciativa o DCC tem mantido um olhar atento sobre o seu princípio fundador, que é compartilhar o acesso a recursos de pesquisa digital e a problemas característicos da e-Science.

Em "Curation of Laboratory Experimental Data as Part of the Overall Data Lifecycle", Frey (2008) pretende elevar a curadoria para que seja considerada pelo cientista de laboratório como parte de suas boas práticas, e não apenas um procedimento para uns poucos especializados em processos de arquivamento. A curadoria em experimentos é uma solução eficaz para o fornecimento de metadados de alta qualidade, tornando-os reutilizáveis e contribuindo com a ciência.

Para Whyte et al. (2008) "Meeting Curation Challenges in a Neuroimaging Group" o projeto SCARP do Grupo de Neuroimagem, da Divisão de Psiquiatria da Universidade de Edimburgo, tem como objetivo abordar as crescentes necessidades de armazenamento de dados e de curadoria, dadas as capacidades oferecidas pela nova infraestrutura. $\mathrm{O}$ estudo analisa brevemente o contexto político e os desafios atuais para a integração de dados e compartilhamento na área da neuroimagem.

Mudanças na forma como os recursos tornam-se obsoletos também são discutidos e com mais valor devem ser colocados sobre o trabalho dos profissionais da curadoria digital. 
A curadoria nas bibliotecas digitais. De acordo com Madden (2008), “Applying the Digital Curation Lessons Learned from American Memory," no projeto American Memory, lançado em 1995, mais de 700.000 itens digitais descritos em 90 coleções foram adicionados à memória americana nos primeiros cinco anos, incluindo o conteúdo de 23 organizações externas. E lições valiosas sobre todos os níveis de curadoria digital surgiam nesta incursão no trabalho da biblioteca digital. Este artigo concentra-se principalmente nas lições aprendidas sobre a conceituação, criação, recepção e ações de preservação de conteúdo digital, no entanto a curadoria está presente.

Em 2009, o Programa de Preservação Digital da Biblioteca Digital da Califórnia (CDL), segundo Abrams; Cruse e Kunze (2009), esteve envolvido em um processo de reinvenção que envolveu transformação significativa de sua perspectiva e infraestrutura. Isso incluiu uma rearticulação de sua missão em termos de curadoria digital, ao invés de preservação. Ao oposto de uma abordagem orientada, o projeto voltou-se para atividades de curadoria e uma ênfase em serviços, em vez de sistemas. Esta última mudança foi motivada por um desejo de depreciar a centralidade do repositório. Tendo o repositório como o locus para a atividade de curadoria que resultou na implantação de um sistema monolítico, um pouco pesado e que ficava aquém dos objetivos desejados para a capacidade de resposta à evolução das necessidades do usuário, à rapidez, e sustentabilidade operacional e administrativa. O Programa esteve buscando um novo rumo para o ambiente de curadoria.

Pesquisadores gregos liderados por Constantopoulos et al. (2009), em "DCC\&U: An Extended Digital Curation Lifecycle Model," afirmam que curadoria digital surgiu como uma nova prática interdisciplinar que visa estabelecer diretrizes para o gerenciamento disciplinado de informação. Eles revisaram dois modelos recentes para curadoria digital introduzidos pelo Centro de Curadoria Digital (DCC) e da Unidade de Curadoria Digital (DCU) do Centro de Investigação Athena. Eles propuseram uma fusão dos dois modelos destacando a necessidade de entender o ciclo de vida da curadoria digital adicionando a) espaços para o registro da experiência de uso, b) um estágio para o aprimoramento do conhecimento e c) vocabulários controlados para denotar conceitos, propriedades e relações. O objetivo das ações propostas é duplo: I) proporcionar um modelo do ciclo de vida mais completo para o domínio curadoria digital e II) fornecer um estímulo para uma discussão mais ampla sobre a agenda de pesquisa.

Desenvolvimentos recentes na indústria de armazenamento resultaram na criação de aplicativos e ferramentas para curadores digitais. Dentre alguns dos citados no periódico, destaca- 
se Todd (2009), que relata como o método de acesso eXtensible, a API XAM centra-se na criação e gestão de informações de referência (também conhecido como conteúdo fixo).

Pryor e Donnelly (2009), em "Skilling Up to Do Data: Whose Role, Whose Responsibility, Whose Career?" Quais as funções necessárias para o gerenciamento eficaz de dados e que tipos de conhecimentos são necessários para os pesquisadores e especialistas de dados que estão exercendo essas funções, parecem discussões bem atuais. Essas perguntas foram feitas em um workshop para criadores e curadores de dados, para identificar as necessidades de formação e oportunidades de carreira para quem trabalha no gerenciamento de dados. Este artigo revisita investigações anteriores sobre os papéis e as responsabilidades exigidas para uma "data workforce".

Meios de informação emergentes apresentam novos desafios para os curadores. Shah (2009) identificou em seu artigo uma série de questões relacionadas com esses objetos digitais, especificamente de vídeos digitais. Resumiu as lições aprendidas proporcionando implicações para os curadores da biblioteca digital interessados em coletar e preservar objetos digitais de natureza efêmera.

A curadoria de dados de investigação científica em universidades norte-americanas, segundo Walters (2009), é uma história de indivíduos empreendedores e do progresso incremental. Um pequeno número de bibliotecas e centros de dados que veem as possibilidades de se tornar "centros de gestão de informação digital," estão tomando medidas empreendedoras para estender além de seus ativos de informação tradicionais a inclusão da gestão de dados de pesquisas científicas e acadêmicas. O Georgia Institute of Technology tem tido um caminho de desenvolvimento em direção a um programa de curadoria de dados com base em sua biblioteca. Walters (2009) aborda ainda alguns aspectos do programa e o contexto, como as parcerias inter-institucionais que promovem o programa de curadoria da biblioteca, as parcerias com as comunidades de pesquisa do campus, e uma proposta de modelo para o desenvolvimento de programas de curadoria. Concluise que, apesar da clara necessidade de curadoria de dados apresentados por pesquisadores tais como os grupos de neurocientistas e biocientistas referenciados neste trabalho, a experiência universitária examinada sugere que a coleta de recursos para o desenvolvimento de programas decuradoria de dados a nível institucional, está provando ser um bem oneroso. No entanto, e apesar dos desafios, algumas universidades de pesquisa dos Estados Unidos estão começando a estabelecer programas de curadoria de dados. 
Witt et al. (2009) em "Constructing Data Curation Profiles" apresentam os métodos, a estruturação (design) e a construção do perfil para a curadoria de dados. Um instrumento que pode ser usado para fornecer informações detalhadas de dados específicos para a curadoria numa biblioteca acadêmica. Os perfis também representam as necessidades de curadoria de dados a partir da perspectiva dos produtores de dados, em sua própria língua. Como tal, eles apoiam a exploração de curadoria de dados em diferentes domínios de investigação, em termos reais e práticos. Com o patrocínio do Institute of Museum and Library Services, os pesquisadores da Purdue University e a Universityof Illinois entrevistaram 19 indivíduos do corpo docente para identificar necessidades de descoberta, acesso, preservação e reutilização de seus dados de pesquisa. Cada perfil apresenta também um conjunto de dados específicos fornecido pelo sujeito para servir como um exemplo concreto. Os perfis estão sendo publicados em uma wiki pública para perguntas e discussão, e, um modelo em branco é utilizado para divulgar as diretrizes para que outros pesquisadores possam criar e compartilhar seus próprios perfis. Este estudo foi realizado principalmente do ponto de vista dos bibliotecários em interação com os professores pesquisadores. No entanto, espera-se que estes resultados complementem uma ampla variedade de investigação e prática da curadoria de dados fora da Biblioteconomia e do ambiente universitário.

A exemplo de outros anos, é importante destacar o simpósio DigCCurr (Curriculum Symposium Digital Curaduration) de 2009, realizado em Chapel Hill, Carolina do Norte, com o tema "Prática da Curadoria Digital, Promessas e Perspectivas". De acordo com Ball e Day (2009) o Simpósio contou com sessões que tratam de questões de vanguarda na investigação sobre curadoria digitale exibiu os recentes desenvolvimentos em ferramentas de curadoria digital. Ao mesmo tempo, o Simpósio também tratou de como formar a nova geração de profissionais da informação com as habilidades necessárias para pesquisa e desenvolvimento prático.

Nas edições de 2010, a ênfase recai na curadoria digital associada à preservação digital e em alguns casos também à mineração de dados. Ainda há um relato importante, sobre os organismos de financiamento que exigem cada vez mais dos pesquisadores a elaboração de planos de gestão de dados (DMPs). O Centro de Curadoria Digital (DCC) criou o DMP online, uma ferramenta na Web que se baseia em uma análise dos requisitos dos financiadores e que permite aos investigadores criar e exportar DMPs personalizáveis, tanto na fase de pedido de subvenção quanto durante a vida do projeto. 
Nas publicações de 2011 os artigos estão vinculados ao dilúvio de dados; os dados científicos devem ser compartilhados para uso além dos fins para que foram inicialmente recolhidos, ou seja o re-uso de dados científicos; uso de ontologias para a integração e curadoria de dados; educação para profissionais de e-Science: integrando curadoria de dados e cyberinfrastructure; fazendo da curadoria digital uma função institucional sistemática; curadoria de dados de pesquisa científica para longo prazo, ou seja, um método de análise de preservação em contexto.

No entanto, o destaque é mais uma vez para um artigo de Higgins (2011), "Digital Curation: The Emergence of a New Discipline". Em meados de 1990, no Reino Unido, a atividade de preservação digital concentrou-se em assegurar a sobrevivência do material digital - estimulado pelo relatório dos EUA sobre a preservação da informação e desenvolvida por meio de atividades financiadas pelo JISC. A curadoria digital surgiu como uma nova disciplina apoiada através das atividades do Centro Digital de Curadoria do Reino Unido e um número de 6 projetos dentro da UE. Agora a curadoria digital está incorporada na prática e na pesquisa; com o desenvolvimento de ferramentas e a fundação de uma série de unidades de apoio a educadores acadêmicos oferecendo treinamento e a promoção de pesquisas.

Em 2012, as edições e artigos estão voltados aos diferentes tipos de conhecimento em dados de pesquisa. Para compreender o 'intensivo' da pesquisa de dados:

- fluxos de dados;

- contexto de strings para arquivos binários;

- dados de classe científica - dados de citação e de publicação de centros de dados ambientais do NERC;

- revisão da evolução das políticas de gestão de dados;

- a desconstrução do ciclo de vida de pesquisa institucional e um portfólio de dez serviços de suporte de dados que as bibliotecas podem oferecer para apoiar as fases do ciclo de vida da pesquisa;

- discussão de formatos de armazenamento de arquivo a partir do ponto de vista da curadoria digital e preservação.

Ainda, a gestão de dados e planejamento para a preservação da Big Science, requer desenvolver um conjunto de ferramentas para fornecer orientações sobre a aplicação das melhores práticas de planejamento dentro de grandes projetos científicos, parece ser o desafio emergente. 
Já 2013 surpreende com um artigo que há tempos era aguardado pela comunidade, as competências requeridas para o profissional de curadoria. Os autores Kim, Warga, e Moen (2013), em seu artigo, “Competencies Required for Digital Curation: An Analysis of Job Advertisements, auxiliam a preencher esta lacuna. Segundo esses autores, com papel cada vez mais importante de curadoria digital no ambiente de informação de dados, há uma necessidade de identificar um conjunto de competências para profissionais da área em crescimento. Como parte de um projeto de desenvolvimento de currículo, financiado pelo Instituto dos EUA do Museumand Library Services, um total de 173 anúncios de emprego publicados entre outubro de 2011 e abril de 2012 foram coletados de diversas fontes. Levaram em conta diversos tipos de profissionais na área de curadoria digital em toda a América do Norte. Título, tipos de instituições e localização, formação educacional, experiência, conhecimentos e habilidades e os deveres foram examinados e analisados. Os resultados da análise mostram que os trabalhos de curadoria digital são caracterizados por uma complexa interação de várias habilidades e conhecimentos. Os resultados deste estudo apresentam exigências emergentes para uma mão de obra qualificada na área de curadoria digital.

Definir o que importa e quando preservar é um dos grandes desafios da curadoria. Yoon (2013) traz um interessante artigo sobre o conteúdo e as coleções digitais pessoais na Web, "Defining What Matters When Preserving Web-Based Personal Digital Collections: Listening to Bloggers". Relata que tais coleções, muitas vezes, contêm memórias pessoais, atividades, pensamentos e até mesmo perfis. Com o aumento na criação de materiais pessoais na Web, as necessidades de arquivamento e preservação desses materiais estão aumentando, não apenas para o propósito de desenvolver arquivos pessoais, mas também com o propósito de capturar a memória social e rastreamento de vestígios humanos nesta época.

Já a vinculação de dados de publicações, rumo a uma abordagem interdisciplinar, Hoogerwerf et al. (2013), enfrentam o desafio de articular informações acadêmicas em infraestrutura de pesquisa multidisciplinares. Há uma tendência para ligar publicações com dados de pesquisa e outras informações, mas, como ainda é um tema emergente, isso é tratado de forma diferente por várias iniciativas e disciplinas.

Para Lagoze et al. (2013), em “Data Management of Confidential Data” levantam uma questão muito importante, onde pesquisadores, principalmente das ciências sociais cada vez mais fazem uso de dados que são confidenciais, que contém ligações com as identidades de pessoas, 
empresas, etc. A confidencialidade dos dados é uma barreira para a sua utilidade e curadoria, tornando-se difícil a gestão de dados e interferindo com as práticas acadêmicas básicas, tais como validação e reutilização de resultados existentes. Descreve-se a complexidade das relações entre os dados que abrangem uma divisão pública e privada. Também descrevem um protótipo, um primeiro passo em proporcionar aos investigadores uma ferramenta que se estende por esta divisão e tornar possível pesquisar, acessar e citar esses dados.

E por fim, no artigo de Kelly et al. (2013), a necessidade crítica de profissionais para curadoria de dados científicos foi o impulso para o programa Data Curation Education in Research Centers (DCERC). Esse está desenvolvendo um modelo sustentável e transferível, para educar alunos de Biblioteconomia e Ciência da Informação, em curadoria de dados, por meio de experiências de campo em centros de pesquisa e de dados. DCERC criou e implementou um programa de pesquisa levando os alunos para o mundo real de curadoria de dados científicos, onde eles se envolvem com as práticas e os desafios atuais e partilham a sua experiência em desenvolvimento e pesquisa. As instituições parceiras estão desenvolvendo e avaliando este modelo com a intenção de ampliar o programa para um quadro maior de parceiros e participantes. Este artigo relata os progressos nas fases iniciais do desenvolvimento do modelo.

Terminamos assim, a análise dos principais resumos selecionados. Mas isso não representa que terminam as reflexões sobre o conteúdo colhido.

\section{CONSIDERAÇÕES FINAIS}

Ao finalizar esse estudo, podemos considerar que um esforço significativo ainda precisa ser empreendido no desenvolvimento de uma infraestrutura para materiais digitais, assim como no desenvolvimento das habilidades requeridas para a curadoria digital para pesquisadores e profissionais da informação. Sem isso, o investimento atual em digitalização, conteúdos digitais, formação de portais e repositórios, só irá garantir benefícios em curto prazo, protelando os benefícios duradouros.

Sobre a nossa questão de pesquisa, o que tem sido escrito sobre a curadoria digital num periódico científico ao longo do tempo, ficou evidente que a curadoria digital se apresenta como 
uma nova área de prática e de pesquisa que dialoga com várias disciplinas e muitos gêneros de profissionais. Identificamos que o estado atual do conceito de curadoria digital, no periódico International Journal of Digital Curation, está associado a outros termos como: curadoria de dados, curadoria de dados digitais, e acima de tudo está atrelado ao ciclo da preservação digital, ao reuso de dados científicos, ao fornecimento de metadados de alta qualidade, ao compartilhamento de dados e a melhoraria da ciência. Sendo ainda difícil estabelecer um conceito único para o termo.

Em nenhum momento identificamos a curadoria digital alinhada à curadoria de informação, serviço que poderia estar atrelado a bibliotecas digitais, ou seja, o papel do profissional que fornece conteúdo a um público específico dentro de um contexto requerido pelo pesquisador ou usuário.

No entanto, nossos objetivos foram alcançados, conseguimos vislumbrar um pouco mais desse cenário promissor. Esses pontos nos inspiram a propor novos produtos e serviços dentro do domínio da Ciência da Informação.

\section{REFERÊNCIAS}

BARDIN, L. Análise de Conteúdo. Lisboa, Portugal: Edições 70, 1977.

BUFREM, Leilah Santiago et al. Produção científica em Ciência da Informação: análise temática em artigos de revistas brasileiras. Perspectivas em Ciência da Informação, Belo Horionte, v. 12, n. 1, p.38-49, 2007. Disponível em:

$<$ http://portaldeperiodicos.eci.ufmg.br/index.php/pci/article/view/248>. Acesso em: 05 jul. 2014.

DIGITAL CURATION CENTRE. Disponível em: < http://www.dcc.ac.uk/digital-curation/whypreserve-digital-data>. Acesso em: 03 jun. 2014.

INTERNATIONAL JOURNAL OF DIGITAL CURATION.Edinburgh (UK): University Of Edinburgh, 2014. Semestral. Disponível em: $<$ http://www.ijdc.net/index.php/ijdc/index $>$. Acesso em: 16 jun. 2014.

MINAYO, M. C. S., O Desafio do Conhecimento: Pesquisa Qualitativa em Saúde. São Paulo: Editora Hucitec/Rio de Janeiro: ABRASCO. 1998.

RICHARDSON, Roberto. Jarry. Pesquisa social: métodos e técnicas. 3. ed. São Paulo: Atlas, 1999.

SAYÃO, Luis Fernando; SALES, Luana Farias. Curadoria digital: um novo patamar para preservação de dados digitais de pesquisa. Informação \& Sociedade: Estudos, v.22, n.3, p. 179- 
191, set./dez. 2012. Disponível em:

<http://www.ies.ufpb.br/ojs/index.php/ies/article/view/12224/8586>. Acesso em: 23 fev. 2013.

UNIVERSIDADE DE SÃO PAULO. Sistema Integrado de Bibliotecas. Relatório de Gestão: exercício SIBiUSP 2010-2013. Organizado por Sueli Mara S. P. Ferreira. São Paulo: SIBiUSP; 2014. Disponível em:

http://eprints.rclis.org/22733/1/RELATORIO\%20SIBiUSP\%20BAIXA.pdf. Acesso em 25 abr 2014.

\section{RESUMOS CITADOS}

ABRAMS, Stephen; CRUSE, Patricia; KUNZE, John.Preservation Is Not a Place.

International Journal Of Digital Curation, Edinburgh (UK), v. 4, n. 1, p.8-21, 2009. Semestral. Disponível em: < http://dx.doi.org/10.2218/ijdc.v4i1.72>. Acesso em: 9 jul. 2014.

BALL , Alexander; DAY, Michael.Report from the Digital Curation Curriculum Symposium (DigCCurr) 2009. International Journal Of Digital Curation, Edinburgh (UK), v. 4, n. 1, p.138151, 2009. Semestral. Disponível em: <http://dx.doi.org/10.2218/ijdc.v4i1.84>. Acesso em: 10 jul. 2014.

BEAGRIE, Neil. Digital Curation for Science, Digital Libraries, and Individuals. International Journal Of Digital Curation, Edinburgh (UK), v. 1, n. 1, p.3-16, jan. 2006. Semestral.

Disponível em: <http://www.ijdc.net/index.php/ijdc/article/view/6>. Acessoem: 1 jul. 2014

CHOUDHURY, Sayeed et al. Digital Data Preservation for Scholarly Publications in Astronomy. International Journal Of Digital Curation, Edinburgh (UK), v. 2, n. 2, p.20-30, jul. 2007. Semestral. Disponível em: <http://dx.doi.org/10.2218/ijdc.v2i2.26>. Acesso em: 9 jul. 2014.

CONSTANTOPOULOS, Panos, etal. DCC\&U: An Extended Digital Curation Lifecycle Model. International Journal Of Digital Curation, Edinburgh (UK), v. 4, n. 1, p.34-45, jan. 2009. Semestral. Disponível em: < http://dx.doi.org/10.2218/ijdc.v4i1.76>. Acesso em: 15 jul. 2014.

DAY, Michael. Report from the DigCCurr 2007 International Symposium on Digital Curation, Chapel Hill, NC, April 18-20, 2007. International Journal Of Digital Curation, Edinburgh (UK), v. 2, n. 1, p.102-111, 2007. Semestral. Disponível em: < http://dx.doi.org/10.2218/ijdc.v2i1.17>. Acesso em: 9 jul. 2014.

FREY, Jeremy. Curation of Laboratory Experimental Data as Part of the Overall Data Lifecycle. International Journal Of Digital Curation, Edinburgh (UK), v. 1, n. 3, p.44-62, jan. 2008. Semestral. Disponível em: <http://www.ijdc.net/index.php/ijdc/article/view/62>. Acessoem: 20 jun. 2014. 
HIGGINS, Sarah. Draft DCC Curation Lifecycle Model. International Journal Of Digital Curation, Edinburgh (UK), v. 1, n. 2, p.82-87, jul. 2007. Disponível em: $<$ http://www.ijdc.net/index.php/ijdc/article/view/46>. Acessoem: 20 jun. 2014.

HIGGINS, Sarah. Digital Curation: The Emergence of a New Discipline. International Journal Of Digital Curation, Edinburgh (UK), v. 2, n. 6, p.78-88, jul. 2011. Semestral. Disponível em: $<$ http://www.ijdc.net/index.php/ijdc/article/view/184>. Acessoem: 30 jun. 2014.

HIGGINS, Sarah. The DCC Curation Lifecycle Model. International Journal Of Digital Curation, Edinburgh (UK), v. 1, n. 3, p.134-140, jan. 2008. Semestral. Disponível em: <http://www.ijdc.net/index.php/ijdc/article/view/69>. Acessoem: 20 jun. 2014.

HOOGERWERF, Mathias Lösch, etal. Linking Data and Publications: Towards a CrossDisciplinary Approach. International Journal Of Digital Curation, Edinburgh (UK), v. 8, n. 1, p.244-254, 2013. Semestral. Disponível em: <http://dx.doi.org/10.2218/ijdc.v8i1.257>. Acesso em: 15 jul. 2014.

KELLY, Karon, etal. Model Development for Scientific Data Curation Education. International Journal Of Digital Curation, Edinburgh (UK), v. 8, n. 1, p.255-264, 2013. Semestral. Disponível em: <http://dx.doi.org/10.2218/ijdc.v8i1.258>. Acesso em: 15 jul. 2014.

KIM, Jeonghyun; WARGA, Edward; MOEN, William E.. Competencies Required for Digital Curation: An Analysis of Job Advertisements. International Journal Of Digital Curation, Edinburgh (UK), v. 1, n. 8, p.66-86. Disponível em: <http://www.ijdc.net/index.php/ijdc/index>. Acessoem: 10 jul. 2014.

LAGOZE, Carl, etal. Data Management of Confidential Data. International Journal Of Digital Curation, Edinburgh (UK), v. 8, n. 1, p.265-278, 2013. Semestral. Disponível em: $<$ http://dx.doi.org/10.2218/ijdc.v8i1.259>. Acesso em: 15 jul. 2014.

LAMBERT, Paul et al. Data Curation Standards and Social Science Occupational Information Resources. International Journal Of Digital Curation, Edinburgh (UK), v. 1, n. 2, p.73-91. 2007. Semestral. Disponível em: <http://www.ijdc.net/index.php/ijdc/article/view/26>. Acessoem: 20 jun. 2014.

MADDEN, Liz. Applying the Digital Curation Lessons Learned from American Memory. International Journal Of Digital Curation, Edinburgh (UK), v. 3, n. 1, p.121-129, 2008. Semestral. Disponível em: <http://dx.doi.org/10.2218/ijdc.v3i2.63 >. Acesso em: 11 jul. 2014.

PRYOR, Graham. Some Challenges for eScience Liaison. International Journal Of Digital Curation, Edinburgh (UK), v. 2, n. 2, p.105-110, jul. 2007. Semestral. Disponível em: $<$ http://dx.doi.org/10.2218/ijdc.v2i2.33> Acesso em: 20 jun. 2014. 
PRYOR , Graham; DONNELLY, Marti. Skilling Up to Do Data: Whose Role, Whose Responsibility, Whose Career? International Journal Of Digital Curation, Edinburgh (UK), v. 4, n. 2, p.158-170, 2009. Semestral. Disponível em: <http://dx.doi.org/10.2218/ijdc.v4i2.105>. Acesso em: 9 jul. 2014.

SHAH, Chirag. Context Miner: Supporting the Mining of Contextual Information for Ephemeral Digital Video Preservation. International Journal Of Digital Curation, Edinburgh (UK), v. 4, n. 2 , p.171-183, 2009. Semestral. Disponível em: <http://dx.doi.org/10.2218/ijdc.v4i2.106>. Acesso em: 10 jul. 2014.

WALTERS, Tyler O. Data Curation Program Development in U.S. Universities: The Georgia Institute of Technology Example. International Journal Of Digital Curation, Edinburgh (UK), v. 4, n. 3, p.83-92, 2009. Semestral. Disponível em: <http://dx.doi.org/10.2218/ijdc.v4i3.116>. Acesso em: 10 jul. 2014.

WHYTE, Angus, et al. Meeting Curation Challenges in a Neuroimaging Group. International Journal Of Digital Curation, Edinburgh (UK), v. 3, n. 1, p.171-181, jan. 2008. Semestral. Disponível em: < http://dx.doi.org/10.2218/ijdc.v3i1.53>. Acesso em: 9 jul. 2014.

WITT, Michael, et al. Constructing Data Curation Profiles. International Journal Of Digital Curation, Edinburgh (UK), v. 4, n. 3, p.93-103, 2009. Semestral. Disponível em:

$<$ http://dx.doi.org/10.2218/ijdc.v4i3.117>. Acesso em: 15 jul. 2014.

TODD, Steve. The eXtensible Access Method (XAM) Standard. International Journal Of Digital Curation, Edinburgh (UK), v. 4, n. 2, p.107-118, 2009. Semestral. Disponível em: < http://dx.doi.org/10.2218/ijdc.v4i2.97>. Acesso em: 10 jul. 2014.

YOON, Ayoung.Defining What Matters When Preserving Web-Based Personal Digital Collections: Listening to Bloggers. International Journal Of Digital Curation, Edinburgh (UK), v. 8, n. 1, p.173-192, 2013. Semestral. Disponível em: <http://dx.doi.org/10.2218/ijdc.v8i1.240>. Acesso em: 10 jul. 2014. 